\title{
Bienvenue à Bruxelles du 8 au 10 avril 2015 ! Participez au $5{ }^{\text {ème }}$ Congrès international francophone de pédagogie des sciences de la santé
}

En tant que société savante, la Société internationale francophone d'éducation médicale (SIFEM) est le lieu de rencontre privilégié de tous les acteurs impliqués dans la formation des professionnels de la santé, qui sont intéressés par les différents aspects de la pédagogie appliquée à ce champ. À cette fin, elle favorise les échanges entre chercheurs, experts, formateurs en éducation, enseignants et praticiens, soucieux de transposer différentes innovations didactiques ou pédagogiques dans leurs milieux.

Le prochain rendez-vous à Bruxelles, du 8 au 10 avril prochain, à l'occasion du $5^{\text {ème }}$ Congrès international francophone de pédagogie des sciences de la santé, entend répondre aux attentes les plus exigeantes des uns et des autres. Il proposera à cet égard une variété de ressources originales, concernant autant les réflexions actuelles relatives aux grands courants conceptuels et scientifiques, que les initiatives opérationnelles et concrètes, développées dans les différents milieux institutionnels et professionnels.

Deux thèmes principaux ont été proposés par le comité d'organisation et par le comité scientifique. Ils concernent respectivement la problématique de la responsabilité sociale des institutions de formation en santé, et la perspective de l'approche pédagogique par compétences en formation des professionnels de la santé. Développés de façon souvent distincte, ces deux champs de réflexion s'avèrent en réalité étroitement interdépendants et convergents.

L'approche par compétences répond d'abord au projet de reformuler les finalités d'une formation, en termes de compétences attendues comme résultat explicite du programme. Elle invite ainsi à réfléchir aux orientations à privilégier pour les dispositifs curriculaires et pédagogiques, dans le cadre d'un questionnement de ces finalités en dialogue avec le monde professionnel et les responsables des politiques de santé. L'approche par compétences invite par ailleurs à exploiter les acquis les plus récents des sciences de l'apprentissage, de manière à mettre en place les dispositifs les plus efficaces et les plus motivants, pour optimiser les résultats et l'impact des formations proposées. Elle tente ainsi de dépasser certaines limites reconnues aux approches conventionnelles, en lien avec un cadre conceptuel élargi aux besoins sociétaux, sociaux et professionnels.

De son côté, l'exigence de responsabilité sociale des institutions de formation des professionnels de santé, récemment réaffirmée dans le cadre d'un consensus mondial, invite également à requestionner en profondeur les finalités et l'architecture des curriculums de formation, un siècle après la publication du rapport Flexner. Elle engage les institutions de formation à devenir des partenaires critiques et proactifs des institutions de soin et des décideurs en santé, en optimisant l'adéquation des professionnels formés aux nécessités des populations qu'ils auront à desservir.

Ainsi, dans le contexte de l'exigence désormais assignée aux différentes institutions de formation des professionnels de la santé de prendre conscience de leur responsabilité sociale, le courant de l'approche pédagogique par compétences pourrait 
apparaître, dans sa dimension à la fois didactique et pédagogique, comme un cadre conceptuel et opérationnel à privilégier. Des conditions sont cependant à examiner dans une perspective critique, pour qu'il favorise une perspective authentiquement émancipatrice de l'action en santé, grâce au levier éducatif et à une meilleure réponse aux besoins des personnes et des communautés. Par ailleurs, dans le cadre du courant de la responsabilité sociale, il s'agira de s'efforcer de documenter la pertinence et la viabilité des démarches de qualité qu'il inspire, et d'en démontrer l'utilité comme stratégie de choix pour améliorer l'impact sur la santé dans des facultés de médecine, de manière à orienter de futurs normes et mécanismes d'accréditation.

Les différentes questions relatives aux enjeux et conditions à mettre en place pour un développement judicieux et synergique de l'approche par compétences et de la perspective de la responsabilité sociale dans les institutions de formation en santé seront ainsi discutées au cours du congrès de Bruxelles.

De nombreux autres thèmes $y$ seront aussi abordés, en lien avec les partenaires institutionnels de la SIFEM et des différents groupes de travail qui ont été mis en place, et qui se réuniront à cette occasion. Chercheurs, enseignants ou responsables institutionnels y partageront leurs expériences et leurs questions sur les grands enjeux et thèmes de la pédagogie moderne : formation à la relation et à la communication, éthique de la recherche en éducation médicale, évaluation des apprentissages, évaluation des enseignements, simulation, etc. Toutes ces questions et réflexions feront l'objet d'éclairages critiques, dans le cadre de format variés (sessions plénières, tables rondes, ateliers, communications orales ou affichées).

Venez partager vos questions et confronter vos points de vue, et faire état de vos expériences.

Nous vous attendons très nombreux à Bruxelles.

Jean-François DENEF Président du congrès

Dominique MAILLARD Présidente de la SIFEM 